\title{
Charge Collection and Field Profile Studies of Heavily Irradiated Strip Sensors for the ATLAS Inner Tracker Upgrade
}

K. Hara ${ }^{\mathrm{x}, \mathrm{y}, *}$, P.P. Allport ${ }^{\mathrm{a}}$, M. Baca ${ }^{\mathrm{a}}$, J. Broughton ${ }^{\mathrm{a}}$, A. Chisholmª ${ }^{\mathrm{a}}$ K. Nikolopoulos ${ }^{\mathrm{a}}$, S. Pyatt ${ }^{\mathrm{a}}$, J.P. Thomas $^{\mathrm{a}}$, J.A. Wilson ${ }^{\mathrm{a}}$,

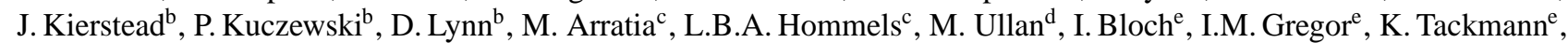

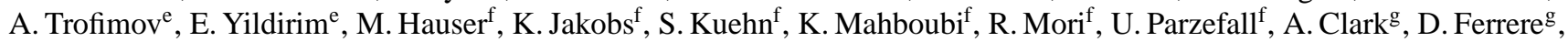
S. Gonzalez Sevilla ${ }^{\mathrm{g}}$, J. Ashby ${ }^{\mathrm{h}}$, A. Blue ${ }^{\mathrm{h}}$, R. Bates ${ }^{\mathrm{h}}$, C. Buttar ${ }^{\mathrm{h}}$, F. Doherty ${ }^{\mathrm{h}}$, T. McMullen ${ }^{\mathrm{h}}$, F. McEwan ${ }^{\text {h }}$, V. O'Shea ${ }^{\text {h }}$, S. Kamada ${ }^{\mathrm{i}}$,

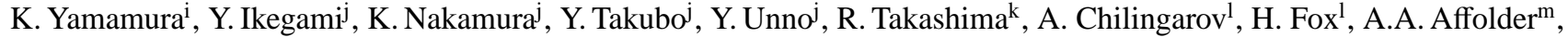

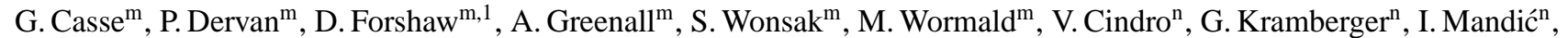

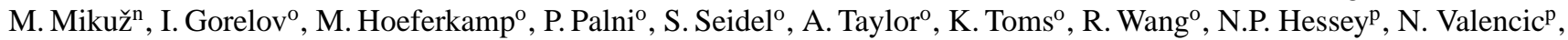
K. Hanagaki ${ }^{\mathrm{g} j}$, Z. Dolezal ${ }^{\mathrm{r}}$, P. Kodys ${ }^{\mathrm{r}}$, J.Bohm ${ }^{\mathrm{s}}$, M.Mikestikova ${ }^{\mathrm{s}}$, A. Bevan ${ }^{\mathrm{t}}$, G. Beck ${ }^{\mathrm{t}}$, C. Milke ${ }^{\mathrm{u}}$, M. Domingo ${ }^{\mathrm{u}}$, V. Fadeyev ${ }^{\mathrm{u}}$, Z. Galloway ${ }^{\mathrm{u}}$, D. Hibbard-Lubow ${ }^{\mathrm{u}}$, Z. Liang ${ }^{\mathrm{u}}$, H. F.-W. Sadrozinski ${ }^{\mathrm{u}}$, A. Seiden ${ }^{\mathrm{u}}$, K. To ${ }^{\mathrm{u}}$, R. French ${ }^{\mathrm{v}}$, P. Hodgson , H. Marin-Reyes ${ }^{\mathrm{v}}$, K. Parker ${ }^{\mathrm{v}}$, O. Jinnouchiw ${ }^{\mathrm{w}}$, K. Hara ${ }^{\mathrm{x}, \mathrm{y}}$, K. Sato ${ }^{\mathrm{x}}$, K. Sato ${ }^{\mathrm{x}, \mathrm{y}}$, M. Hagihara ${ }^{\mathrm{x}}$, S. Iwabuchi ${ }^{\mathrm{x}}$, J. Bernabeu ${ }^{z}$, J.V. Civera ${ }^{\text {z }}$ C. Garcia ${ }^{z}$, C. Lacasta ${ }^{z}$, S. Marti i Garcia ${ }^{z}$, D. Rodriguez ${ }^{z}$, D. Santoyo ${ }^{z}$, C. Solaz , U. Soldevila ${ }^{z}$

\author{
${ }^{a}$ School of Physics and Astronomy, University of Birmingham, Birmingham B15 2TT, United Kingdom \\ ${ }^{b}$ Brookhaven National Laboratory, Physics Department and Instrumentation Division, Upton, NY 11973-5000, USA \\ ${ }^{c}$ Cavendish Laboratory, University of Cambridge, JJ Thomson Avenue, Cambridge CB3 OHE, United Kingdom \\ ${ }^{d}$ Centro Nacional de Microelectronica (IMB-CNM, CSIC), Campus UAB-Bellaterra, 08193 Barcelona, Spain \\ ${ }^{e}$ DESY, Notkestrasse 85, 22607 Hambrug, Germany

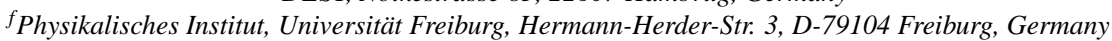 \\ ${ }^{g}$ DPNC, University of Geneva, 24, Quai Ernest-Ansermet, CH-1211 Geneve 4, Switzerland \\ ${ }^{h}$ SUPA-School of Physics and Astronomy, University of Glasgow, Glasgow G12 8QQ, United Kingdom \\ ${ }^{i}$ Solid State Div., Hamamatsu Photonics K.K., 1126-1, Ichino-cho, Higashi-ku, Hamamatsu-shi, Shizuoka 435-8558, Japan \\ ${ }^{j}$ Institute of Particle and Nuclear Study, KEK, Oho 1-1, Tsukuba, Ibaraki 305-0801, Japan \\ ${ }^{k}$ Department of Science Education, Kyoto University of Education, Kyoto 612-8522, Japan \\ ${ }^{l}$ Physics Department, Lancaster University, Lancaster LA1 4YB, United Kingdom \\ ${ }^{m}$ Oliver Lodge Laboratory, Department of Physics, University of Liverpool, Oxford St., Liverpool L69 7ZE, United Kingdom \\ ${ }^{n}$ Jožef Stefan Institute and Department of Physics, University of Ljubljana, Ljubljana, Slovenia \\ ${ }^{\circ}$ Department of Physics and Astronomy, University of New Mexico, MSC07 4220, 1919 Lomas Blvd. NE, Albuquerque, NM 87131, USA \\ ${ }^{p}$ Nikhef, Science Park 105, 1098 XG Amsterdam, Netherlands \\ ${ }^{q}$ Department of Physics, Osaka University, Machikaneyama-cho 1-1, Toyonaka-shi, Osaka 560-0043, Japan \\ ${ }^{r}$ Charles University in Prague, Faculty of Mathematics and Physics, V Holesovickach 2, Prague 8, Czech Republic \\ ${ }^{s}$ Academy of Sciences of the Czech Republic, Institute of Physics, Na Slovance 2, 18221 Prague 8, Czech Republic \\ ${ }^{t}$ School of Physics and Astronomy, Queen Mary University of London, London E1 4NS, United Kingdom \\ ${ }^{u}$ Santa Cruz Institute for Particle Physics (SCIPP), University of California, Santa Cruz, CA 95064, USA \\ ${ }^{v}$ Department of Physics and Astronomy, The University of Sheffield, Hicks Building, Hounsfield Road, S3 7RH Sheffield, United Kingdom \\ ${ }^{w}$ Institute of Science and Engineering, Tokyo Institute of Technology, Ookayama 2-12-1, Meguro-ku, Tokyo 152-8551, Japan \\ ${ }^{x}$ Institute of Pure and Applied Sciences, University of Tsukuba, Tsukuba, Ibaraki 305-8751, Japan \\ ${ }^{y}$ Center for Integrated Research in Fundamental Science and Engineering, University of Tsukuba, Tsukuba, Ibaraki 305-8571 Japan \\ ${ }^{z}$ IFIC/CSIC-UVEG, Ed. Inst. Investigacion, PO Box 22085, 46071 Valencia, Spain
}

\begin{abstract}
The ATLAS group has evaluated the charge collection in silicon microstrip sensors irradiated up to a fluence of $1 \times 10^{16} \mathrm{n}_{\mathrm{eq}} / \mathrm{cm}^{2}$, exceeding the maximum of $1.6 \times 10^{15} \mathrm{n}_{\mathrm{eq}} / \mathrm{cm}^{2}$ expected for the strip tracker during the high luminosity LHC (HL-LHC) period including a safety factor of 2 . The ATLAS12, $\mathrm{n}^{+}$-on-p type sensor, which is fabricated by Hamamatsu Photonics (HPK) on float zone (FZ) substrates, is the latest barrel sensor prototype. The charge collection from the irradiated $1 \times 1 \mathrm{~cm}^{2}$ barrel test sensors has been evaluated systematically using penetrating $\beta$-rays and an Alibava readout system. The data obtained at different measurement sites are compared with each other and with the results obtained from the previous ATLAS07 design. The results are very consistent, in particular, when the deposit charge is normalized by the sensor's active thickness derived from the edge transient current technique (edge-TCT) measurements. The measurements obtained using $\beta$-rays are verified to be consistent with the measurements using an electron beam. The edge-TCT is also effective for evaluating the field profiles across the depth. The differences between the irradiated ATLAS07 and ATLAS12 samples have been examined along with the differences among the samples irradiated with different radiation sources: neutrons, protons, and pions. The studies of the bulk properties of the devices show that the devices can yield a sufficiently large signal for the expected fluence range in the HL-LHC, thereby acting as precision tracking sensors.
\end{abstract}

Keywords: high luminosity Large Hadron Collider, microstrip sensor, charge collection, edge transient current technique, field

profile

April 12, 2016 


\section{Introduction}

The ATLAS experiment [1] at the Large Hadron Collider ${ }_{56}^{55}$ (LHC) is due to undergo phased detector upgrades in accor- ${ }_{57}$ dance with the planned accelerator upgrades. The instantaneous ${ }_{58}^{57}$ beam luminosity after the Phase-II upgrade of the LHC, termed ${ }_{59}^{58}$ the HL-LHC (high luminosity LHC) [2], is expected to reach ${ }_{60}$ $5 \times 10^{34} \mathrm{~cm}^{-2} \mathrm{~s}^{-1}$ and deliver a total of $3000 \mathrm{fb}^{-1}$ of collisions at ${ }_{61}$ a center-of-mass energy of $14 \mathrm{TeV}$. The ATLAS inner detector is subjected to a major upgrade to cope with these significant ${ }_{63}$ increases in the instantaneous and integrated luminosities. A ${ }_{64}$ new all-semiconductor type inner tracker (ITk) [3] [4] [5] composed of pixel and microstrip layers will be installed. As described in the Letter of Intent (LoI) [6] the microstrip detector is constructed of five barrel layers and seven discs in each of the 66 endcaps. The barrel strips in the three inner layers are $24 \mathrm{~mm}{ }^{67}$ long while in the outer layers they are $48 \mathrm{~mm}$ long. The strip pitch is $74.5 \mu \mathrm{m}$ in both cases. The length of the endcap strips ${ }^{68}$ varies from $17 \mathrm{~mm}$ to $59 \mathrm{~mm}$ depending on the radius. The 69 strip length is chosen so as to maintain the average hit occu- 70 pancy at less than $1 \%$ at the expected maximum instantaneous ${ }^{71}$ luminosity.

The particle fluence and total ionizing dose expected at the ${ }^{73}$ end of experiment's lifetime has been evaluated with a FLUKA ${ }^{74}$ simulation [7]: this simulation is based on the experience from ${ }^{75}$ the current detector where the agreement between simulation 76 and measurement is within $20 \%$ [7]. The estimated maximum ${ }^{77}$ lifetime fluence values [7] are $5.3 \times 10^{14} \mathrm{n}_{\mathrm{eq}} / \mathrm{cm}^{2}$ in the $24 \mathrm{~mm}{ }^{78}$ long barrel strips, $2.9 \times 10^{14} \mathrm{n}_{\mathrm{eq}} / \mathrm{cm}^{2}$ in the $48 \mathrm{~mm}$ long barrel strips, and $8.1 \times 10^{14} \mathrm{n}_{\mathrm{eq}} / \mathrm{cm}^{2}$ in the endcap. Here, the con- 79 tributions of different kind of particles are translated into 1- 80 $\mathrm{MeV}$ neutron equivalent values by taking into account the non- ${ }_{81}$ ionizing energy loss (NIEL) [8] factors in silicon. The parti- 82 cle composition $(\mathrm{n}, \pi, \mathrm{p})$ varies according to the location and is ${ }_{83}$ found to be $(57 \%, 35 \%, 8 \%)$ and $(73 \%, 19 \%, 8 \%)$ at the points 84 with the largest fluence values in the $24 \mathrm{~mm}$ and $48 \mathrm{~mm}$ long 85 barrel strips [9].

We have extensively studied the radiation hardness of Hama- 87 matsu $\mathrm{n}^{+}$-on-p float-zone (FZ) strip sensors to predict the per- 88 formance at the end of lifetime and determine if the collected ${ }_{89}$ charge is sufficiently large. Previous studies [10] using the AT- 90 LAS07 layout sensors [11] have shown that after irradiation 91 to the HL-LHC fluence the charge is reduced. However, the ${ }_{92}$ signal-to-noise ratio is more than 15 at $500 \mathrm{~V}$ bias and thus, the ${ }_{93}$ detector remains as a precision tracker.

The ATLAS12A (A12A, for short) and ATLAS12M 95 (A12M)[12] sensor layouts are the latest sensor designs imple- 96 mented at the time of writing (2016). A12 main sensors have 97 the axial strips only and A12M main sensors have the axial and 98 stereo strips mixed. Different bulk resistivity and further de- 99 sign evolution compared to A07 sensors allow us to find ef- ${ }_{-100}$ fects that depend on them and further assure that the resistivity ${ }_{101}$ range expected in production is within the required specification. The bulk damage properties of these series were compared ${ }_{102}$

\footnotetext{
${ }^{*}$ Corresponding author. e-mail: hara@ @ep.px.tsukuba.ac.jp

${ }^{1}$ Now at Syracuse University.
}

with those of the ATLAS07 (A07) series, and the dependence of damage difference on the radiating particle type (neutron, proton, pion) was investigated. The collected charge values were evaluated by seven groups using identical Alibava [13] readout systems utilizing penetrating $\beta$ rays emitted from ${ }^{90} \mathrm{Sr}$. We extended the study to a $4.4-\mathrm{GeV}$ electron beam to cross check if the $\beta$ results are representative of the HL-LHC experiment.

The magnitudes of damage due to neutrons, protons and pions were found to be different for the same NIEL fluence of $5 \times 10^{14} \mathrm{n}_{\mathrm{eq}} / \mathrm{cm}^{2}$. The signal as a function of sensor depth was evaluated using an edge transient current technique (TCT) [14] to determine the field profile after these irradiations.

\section{Samples, Irradiation, and Charge Collection Measure- ments}

\subsection{Samples}

Hamamatsu utilizes 6" wafers for sensor fabrication. The $9.75 \times 9.75 \mathrm{~cm}$ main sensor, which is surrounded by 24 pieces of $1.0 \times 1.0 \mathrm{~cm}$ miniature sensors, is placed on each wafer. Only the miniature sensors are used in this study. The A07, A12A, and A12M sensors are fabricated on the same p-type wafer category FZp, where the resistivity was specified to be within 3 to $8 \mathrm{k} \Omega \mathrm{cm}$. In practice, the typical sensor resistivity varied among the wafer production lots. Consequently, the full-depletion voltages are typically $200-220 \mathrm{~V}(4.3-4.7 \mathrm{k} \Omega \mathrm{cm})$ for $\mathrm{A} 07,220 \mathrm{~V}$ for $\mathrm{A} 12 \mathrm{M}$, and $270-320 \mathrm{~V}(2.9-3.5 \mathrm{k} \Omega \mathrm{cm})$ for $\mathrm{A} 12 \mathrm{~A}$.

\subsection{Irradiation}

The samples were irradiated with neutrons at Ljubljana TRIGA Reactor [15], with $300-\mathrm{MeV}$ pions at PSI, and with 23-, 27-, 70-, and 800-MeV protons at Karlsruhe, Birmingham, CYRIC (Tohoku University, Japan), and Los Alamos accelerators. The $1-\mathrm{MeV}$ neutron equivalent fluence values were calculated using NIEL hardness factors [8].

As an exemplary irradiation setup, the scanning box and sample holder used at CYRIC are shown in the photograph ( Fig. 1). There are fifteen sample slots in the box. The holder with the samples to be irradiated was pushed in remotely, and then the box was moved laterally to allow for uniform irradiation of the samples with the proton beam. The box was thermally insulated and liquid nitrogen was flushed through it to maintain a temperature of approximately $-15^{\circ} \mathrm{C}$. Aluminum foils were attached to the samples to obtain dosimetry using $\mathrm{Al}^{27}(\mathrm{p}, 3 \mathrm{pn}) \mathrm{Na}^{24}$ spallation reaction. For fluences in the range of $10^{12}-10^{16} \mathrm{n}_{\mathrm{eq}} / \mathrm{cm}^{2}$, samples were irradiated from few minutes to six hours at a beam current ranging from $10 \mathrm{nA}$ to $1 \mu \mathrm{A}$. The fluence uncertainty was approximately $10 \%$, as determined from the uncertainty in the available spallation cross section. The irradiated samples were stored in a refrigerator immediately after a series of irradiation was completed.

\subsection{Charge collection measurement technique and calibration}

All the measurements reported here utilized the Alibava system [13] which uses a Beetle analogue readout chip with a fieldprogrammable gate array (FPGA)-based readout. The Alibava 


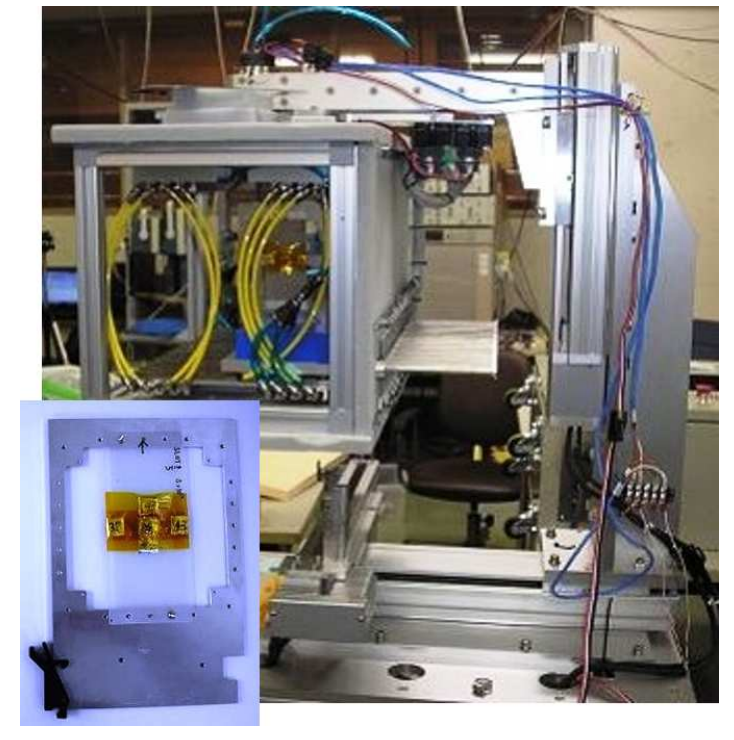

Fig. 1: Sample box used in the irradiation experiment at CYRIC. The bottomleft inset shows the sample holder to be inserted in the sample box.

system digitizes charge while recording the trigger arrival time with respect to the clock cycle. Since a scintillating counter set underneath the sample triggers on the penetrating $\beta$ particles generated through ${ }^{90} \mathrm{Sr}$ decay, the trigger timing is not always optimal with respect to the charge sampling timing. To be se- $^{138}$ lected for the analysis, events were required to be within $5 \mathrm{~ns}^{139}$ of the trigger signal which resulted in the maximum collected ${ }^{140}$ charge. The collected charge of an event was determined by ${ }^{141}$ a clustering algorithm with a seed threshold of 3.5 times the ${ }^{142}$ channel's noise level and neighbor threshold of 1.5 times the $e^{143}$ noise. The collected charge for the sample at a given voltage $e^{144}$ was found by fitting of the cluster charge distribution with $\mathrm{a}^{145}$ Landau function convoluted with a Gaussian. The most prob- ${ }^{146}$ able cluster charge is defined as the collected charge. Instead ${ }^{147}$ of relying on the internal calibration of the Beetle, we have de- ${ }^{148}$ termined the calibration with non-irradiated samples assuming ${ }^{149}$ charge collection $Q\left[\mathrm{e}^{-}\right]$above depletion voltage to be

$$
Q=\frac{d}{3.68}[190+16.3 \ln (d)]
$$

for the measured active thickness $d[\mu \mathrm{m}]$ of the device [16].152 Note that active thickness is typically $10 \mu \mathrm{m}$ smaller than phys- 153 ical thickness, which is explained in Sec. 4.

Figure 2 shows the plot of collected charge vs. bias voltage $e_{155}$ measured for non-irradiated samples and at various measure-156 ment sites. Most of the curves are for A12A samples except ${ }_{157}$ for one corresponding to an A07. As explained previously the ${ }_{158}$ full depletion voltage of A07 is lower than that of the others ${ }_{159}$ but all the samples show identical collected charge above full ${ }_{160}$ depletion.

The average subtracted collected charge is plotted in Fig. ${ }_{162}$ for the A12A sensors. Larger variations are only seen when not ${ }_{163}$ fully depleted: above depletion agreement is very good. Since 164 different sites used different samples, any resistivity and thick-165

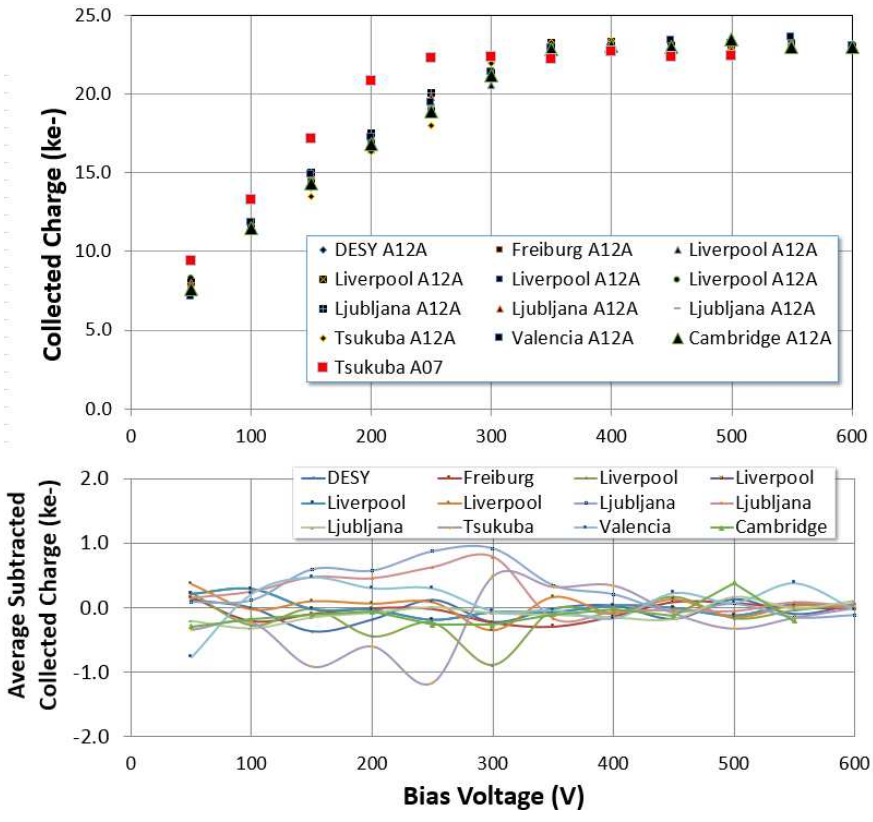

Fig. 2: (top) Collected charge vs. bias for non-irradiated samples measured at different sites. The charge is normalized to $23 \mathrm{ke}^{-}$for a sample with an active thickness of $300 \mu \mathrm{m}$. All samples are A12A except for one A07. (bottom) Average subtracted collected charge in $\mathrm{ke}^{-}$for twelve A12A non-irradiated samples.

ness variations within the production lead to changes in the normalization. Since he observed variation is less than $1 \mathrm{ke}^{-}$below full depletion and at most $0.3 \mathrm{ke}^{-}$above full depletion, these effects are minimal.

The samples were measured at low temperatures, around $-20^{\circ} \mathrm{C}$ to suppress the leakage current induced by radiation. The actual temperatures were different among the measurement sites depending on the cooling system performance. Since the calibration of the Alibava system has a temperature dependence, charge calibration relying on non-irradiated sensors of known thickness, as described, enhances the reliability of charge measurement.

\section{Results of Charge Collection Measurements}

\subsection{Annealing}

The annealing properties of the samples irradiated to $\sim 10^{15}$ $\mathrm{n}_{\mathrm{eq}} / \mathrm{cm}^{2}$ are quite different from those reported previously [17], especially at high bias voltages. Figure 3 shows the collected charge of the A12A and A12M sensors irradiated with protons to a fluence of $10^{15} \mathrm{n}_{\mathrm{eq}} / \mathrm{cm}^{2}$, measured as a function of annealing time at $60^{\circ} \mathrm{C}$. The collected charge increases (beneficial annealing) up to $80 \mathrm{~min}$, and then decreases for both samples at lower bias voltages. However, the collected charge of A12M stays almost constant at bias voltages of $900 \mathrm{~V}$ and $1000 \mathrm{~V}$ after $80 \mathrm{~min}$. Similar trends as for A12M have been observed in another study [18] conducted on A07 sensors, where the samples were irradiated to $10^{15} \mathrm{n}_{\mathrm{eq}} / \mathrm{cm}^{2}$ by neutrons and pions. Owing to radiation-induced defects, the depletion depth decreases with radiation. Since depletion develops from the strip side and 
the applied bias voltage is sustained in the depletion region, the field around the strips increases with irradiation and bias, reaching a region where avalanche multiplication occurs.

The magnitude of initial beneficial annealing is dependent on irradiation conditions such as irradiation rate [19]. Therefore a certain controlled annealing is preferred prior to comparing various types of irradiation data. Also in real experiments where the radiation rate is much smaller and beneficial annealing is taking place, results evaluated with controlled annealing are expected to represent actual characteristics in the real experiments. In the following, the results of charge collection measurement after controlled annealing for $80 \mathrm{~min}$ at $60^{\circ} \mathrm{C}$ are presented.
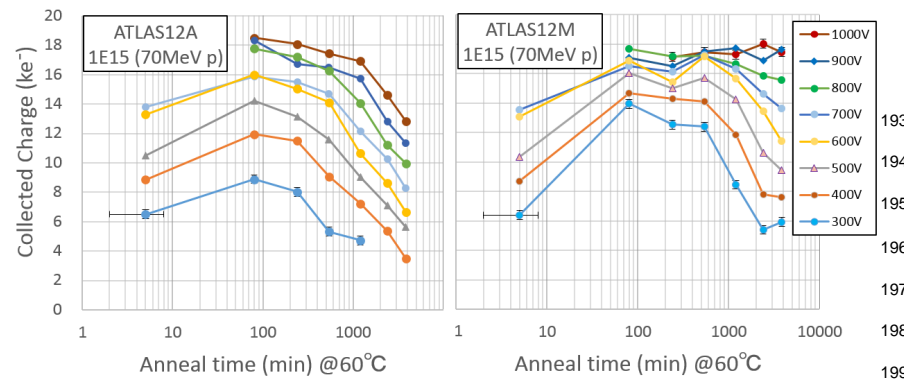

Fig. 3: Examples of annealing properties of A12A (left) and A12M (right). The 200 samples were irradiated with protons to a fluence of $1 \times 10^{15} \mathrm{n}_{\mathrm{eq}} / \mathrm{cm}^{2}$.

\subsection{Neutron and proton irradiation}

Figure 4 shows the collected charge at $500 \mathrm{~V}$ and $900 \mathrm{~V}$ as a function of fluence for all neutron-irradiated A12A and A07 samples. As expected from [20] [21], there is a difference between A12A and A07 sensors observed below $5 \times 10^{14}$ $\mathrm{n}_{\mathrm{eq}} / \mathrm{cm}^{2}$ due to the difference in the initial resistivity of the devices. At higher fluences, this effect is much reduced.

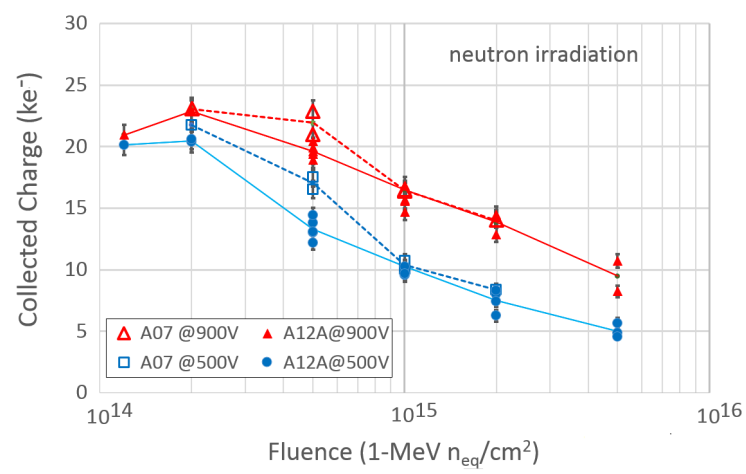

Fig. 4: Collected charge of neutron-irradiated samples measured at $500 \mathrm{~V}$ and $900 \mathrm{~V}$. The lines connect the average data points separately for A07 and A12A samples.

All the data points measured for proton irradiated A12A samples are plotted for three bias voltage settings, 300, 500, and $900 \mathrm{~V}$, in Fig. 5. No noticeable dependence on the source of proton irradiation or the charge collection measurement site was observed for these results.

In Fig. 6, the results of $\mathrm{A} 12 \mathrm{~A}$ are compared with those of $\mathrm{A} 07$ and $\mathrm{A} 12 \mathrm{M}$ at $300 \mathrm{~V}, 500 \mathrm{~V}$, and $900 \mathrm{~V}$. Since the A12A

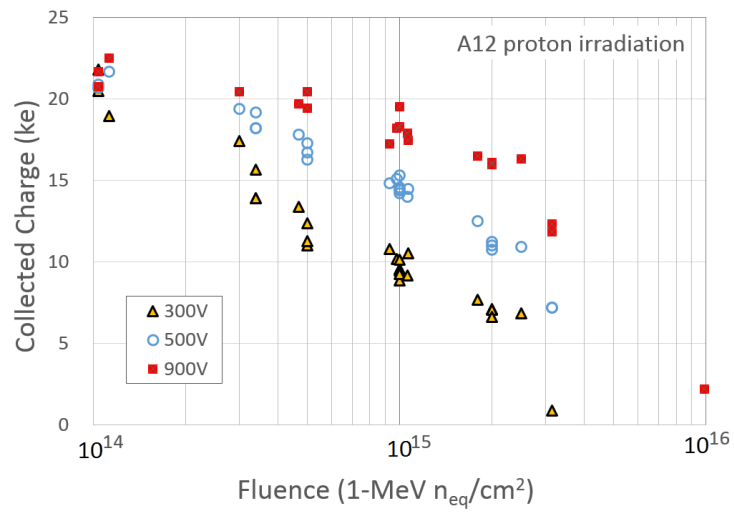

Fig. 5: Collected charge of proton irradiated A12A samples measured at 300 , 500 , and $900 \mathrm{~V}$

data points, as shown in Fig. 5, form specific bands, we take the averages if multiple data points are available, with the variations represented as uncertainty.

At $500 \mathrm{~V}$, the difference among proton irradiated samples is substantially reduced, compared to that observed at $300 \mathrm{~V}$, as expected for the different full depletion voltages. Moreover, the difference to the neutron-irradiated samples becomes small at $900 \mathrm{~V}$ as shown in the figure.

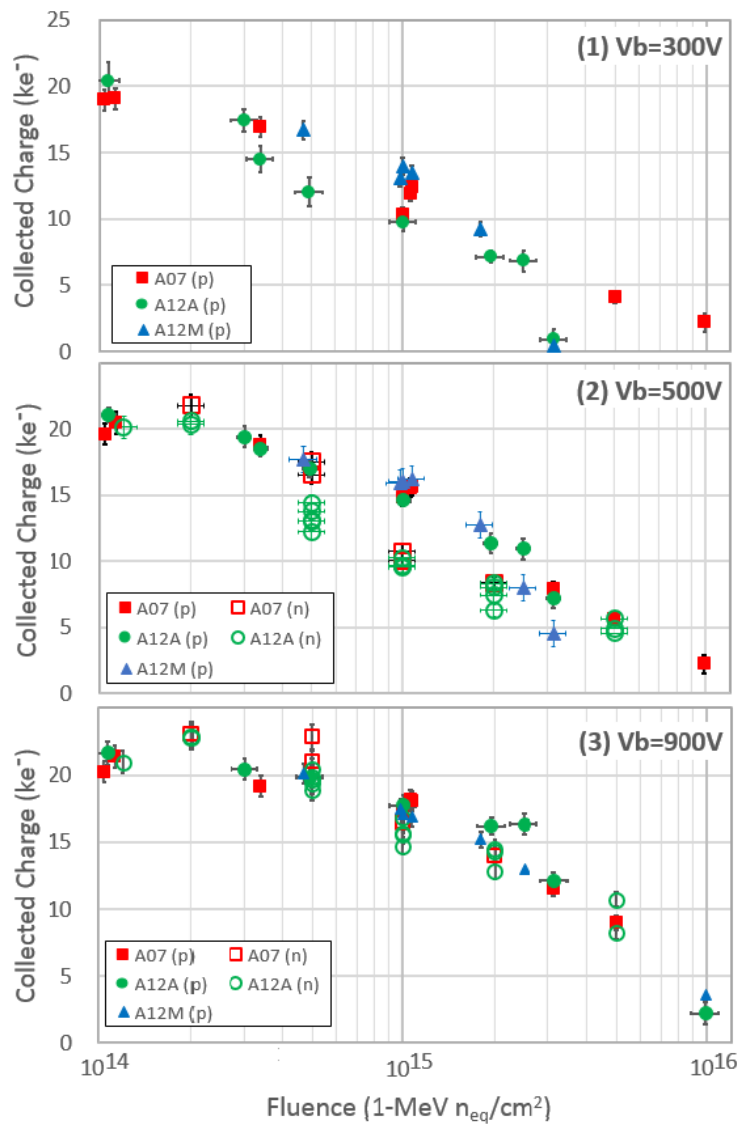

Fig. 6: Comparison of collected charge of proton-irradiated A07, A12A, and A12M samples, measured at (1) $300 \mathrm{~V}$, (2) $500 \mathrm{~V}$, and (3) $900 \mathrm{~V}$. The A12A data points are the averages of the data shown in Fig. 5 with variations represented as uncertainty. The neutron data at $500 \mathrm{~V}$ and $900 \mathrm{~V}$, as shown in Fig. 4, are also plotted. 


\subsection{Test beam evaluation of neutron-irradiated samples}

The charge collection from a few neutron-irradiated samples was measured using a DESY $4.4 \mathrm{GeV}$ electron beam. Two samples, read out with the Alibava system, were placed in the beam in between two beam tracker systems. The samples were cooled to $-25 \pm 3^{\circ} \mathrm{C}$ by circulating cooled Silicone oil around them.

Figure 7 compares the charge distributions of the nonirradiated and $1 \times 10^{15} \mathrm{n}_{\mathrm{eq}} / \mathrm{cm}^{2}$ irradiated samples between the source and beam measurements. The distributions of cluster size, which is determined by the number of hit strips in a hit cluster, are also plotted. The charge distributions are consistent between the source and beam. The cluster size distributions are slightly wider for the source measurement, which can be explained by the incident angle and scattering effects of $\beta$ rays.
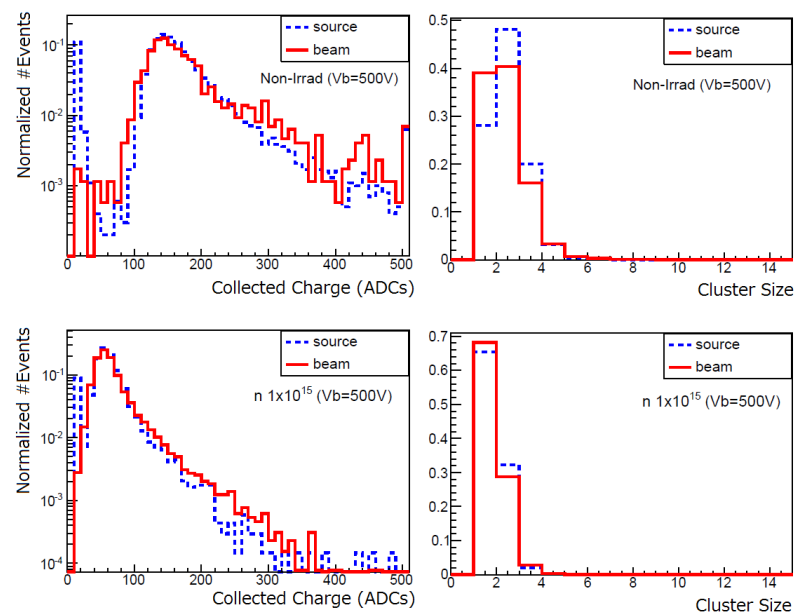

Fig. 7: Comparison of source (dashed) and beam (solid histograms) measurements of the non-irradiated (above) and $1 \times 10^{15} \mathrm{n}_{\mathrm{eq}} / \mathrm{cm}^{2}$ irradiated samples (below). Comparison of charge distributions and cluster size distributions at $500 \mathrm{~V}$.

The most probable charge values are compared in Fig. 8 as ${ }^{236}$ a function of the detector bias for eight samples: two non ${ }^{237}$ irradiated and six neutron-irradiated samples. Of the six sam- ${ }^{238}$ ples, two were measured before being subjected to controlled ${ }^{239}$ annealing. The agreement between the two measurements is ${ }^{240}$ remarkable, although there is a slight tendency for the collected ${ }^{241}$ charge of the irradiated samples for the beam data to be $5-6 \%{ }^{243}$ higher than that for the source data.

The results of this study with the electron beam verified that ${ }^{244}$ measurements using a ${ }^{90} \mathrm{Sr} \beta$ source can provide reliable values ${ }^{245}$ in estimation of collected charge for minimum-ionizing particles.

\subsection{Comparison among proton, neutron, and pion irradiation}

As discussed in Sec. 3.2, the reduction in charge collection ${ }^{250}$ is higher for neutrons than for protons at $500 \mathrm{~V}$ bias. A sys- ${ }^{251}$ tematic comparison of the collected charge was carried out for ${ }^{252}$ the A12A samples of similar initial full depletion voltages ir- $^{253}$ radiated with neutrons, $23-\mathrm{MeV}$ protons (Karlsruhe), and 300-254 $\mathrm{MeV}$ pions (PSI) to the same NIEL fluence of $5 \times 10^{14} \mathrm{n}_{\mathrm{eq}} / \mathrm{cm}^{2} .255$ From Fig. 9, we observe that the collected charge is larger ${ }^{256}$

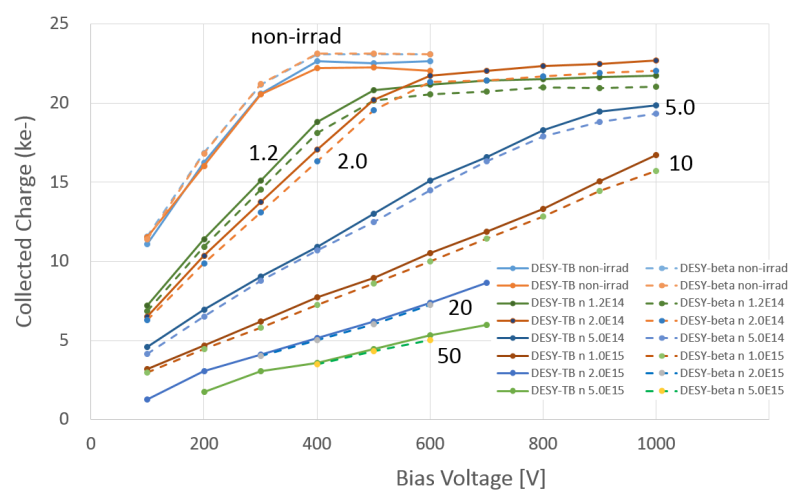

Fig. 8: Comparison of collected charge values between beam (solid line) - and source (dashed line) -measurements, shown as a function of bias. The samples are non-irradiated or irradiated with neutrons to the fluence denoted by the numbers shown next to the curves (the unit is $10^{14} \mathrm{n}_{\mathrm{eq}} / \mathrm{cm}^{2}$ ). The curves 20 and $50 \times 10^{14} \mathrm{n}_{\mathrm{eq}} / \mathrm{cm}^{2}$ are for the data before annealing.

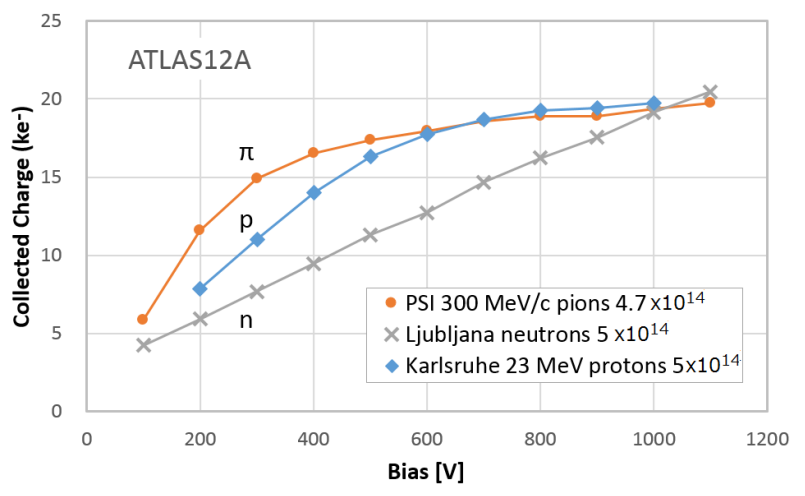

Fig. 9: Collected charge vs. bias of A12A samples irradiated with neutrons, protons, and pions to a fluence of $5 \times 10^{14} \mathrm{n}_{\mathrm{eq}} / \mathrm{cm}^{2}$.

for the pion-irradiated sample than for the proton- or neutronirradiated samples. For a bias of $500 \mathrm{~V}$ or less, the difference is noticeably large.

The results clearly suggest that NIEL hypothesis is not suitable for the normalization of collectable charge at the concerned fluence level, which is typically $1 \times 10^{15} \mathrm{n}_{\mathrm{eq}} / \mathrm{cm}^{2}$.

The differences after irradiation are the main subject of the study presented in Sec. 4 . We discuss the differences in the field profile along the depth instead in the full depletion voltages, since the irradiated sensors do not remain as a simple diode and characterization by the full depletion only is not appropriate.

\subsection{Charge Collection at $500 \mathrm{~V}$}

Figure 10 plots the summary of neutron, proton, and pion irradiation measurements at $500 \mathrm{~V}$ including the measurements using the electron beam. The maximum operation voltage at the HL-LHC, expected in ATLAS, is $500 \mathrm{~V}$, which is determined from the specifications of the available cables used in the present inner detector. At $500 \mathrm{~V}$, all proton-irradiated samples, A07, A12A, and A12M show similar fluence dependences, while the collected charge is reduced for neutron-irradiated samples in the fluence range of $0.5-2 \times 10^{15} \mathrm{n}_{\mathrm{eq}} / \mathrm{cm}^{2}$ for A12A, and of $1-2 \times 10^{15} \mathrm{n}_{\mathrm{eq}} / \mathrm{cm}^{2}$ for A07 samples. 


\section{Field Profile Studies}

In the transient current technique (TCT), transient currents 295 on readout electrodes are induced by charge released in the de-296 tector by short laser pulses. The charge collection characteris-297 tics of the silicon detector can be investigated from the induced ${ }_{298}$ current pulses. The method, when the laser light is injected 299 from the detector side, is effective in evaluating the active thick- 300 ness and understanding the difference between the sensors and $\mathrm{d}_{301}$ irradiation sources.

In an edge-TCT [14] study, an infrared laser of $1060 \mathrm{~nm}_{303}$ wavelength and $300 \mathrm{ps}$ duration was injected from a polished $\mathrm{d}_{304}$ side edge parallel to the strip direction. The induced current 305 from one of the strips was measured using a high-speed am-306 plifier and a $1.5 \mathrm{GHz}$ oscilloscope, in which neighboring strips 307 were set to the same potential as the readout strip to avoid dis-308 turbance of the electric field in the strip sensor. The laser was 309 collimated to $8 \mu \mathrm{m}$ (FWHM) underneath the readout strip. Byз10 scanning through the depth of the sensor the charge collection characteristics can be investigated as a function of sensor depth.

The induced current time profile is influenced by the number of generated charge carriers, carrier velocities, and carrier trapping along their paths. The current profile right after the laser injection can be expressed as [22]:

$$
I(y, t \sim 0) \sim q E_{w}(y)\left[\overline{v_{e}}(y)+\overline{v_{h}}(y)\right]
$$

where $y$ denotes the depth location in the sensor, $E_{w}(y)$ is the weighting field in Ramo's theorem, $q$ is the elementary charge, and $\overline{v_{e}}$ and $\overline{v_{h}}$ are electron and hole velocities, respectively. The time $t \sim 0$ condition in the equation is a result of the require-311 ment that the current amplitude is measured immediately after 312 carrier generation before trapping and before charges move sig-313 nificantly away from the location of the laser beam. When the 314 laser beam is perpendicular to the strips, the weighting field $d_{315}$ is effectively constant because of the contributions of carriers 316 drifting to the neighboring strips [14]. The current values at 317 $t=0.6 \mathrm{~ns}$ was found to be adequate for measurement of the ${ }_{318}$ velocity sum, $\overline{v_{e}}(y)+\overline{v_{h}}(y)[22]$.

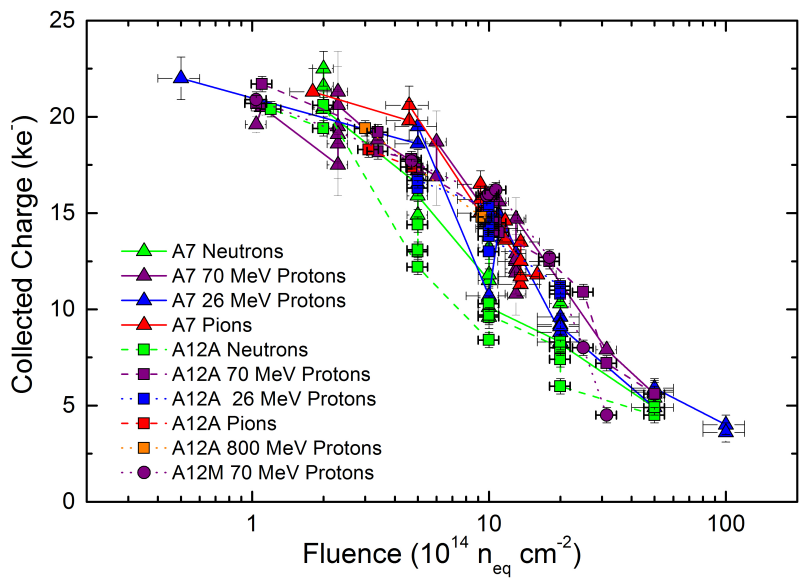

Fig. 10: Summary of collected charge measurements at $500 \mathrm{~V}$ for A07, A12A336 and A12M samples, shown separately for various irradiation sources.
Since velocities are expressed as the product of mobilities and the electric field, the electric field can be extracted simply by using $I(y, t \sim 0) \sim \overline{v_{e}}(y)+\overline{v_{h}}(y)=\left[\mu_{e}(E)+\mu_{h}(E)\right] E$, provided that the electric field is not very high, as velocity saturates with increasing electric field. The detailed analysis has been reported in a previous study [22].

The velocity sum, $\bar{v}_{e}(y)+\overline{v_{h}}(y)$, is plotted in Fig. 11 as a function of sensor depth for non-irradiated A07 and A12A samples at $200 \mathrm{~V}$ and $400 \mathrm{~V}$. Here, the vertical scale is arbitrary but the sample areas in the same plot are normalized, since integration of the electric field $\left(\sim \bar{v}_{e}(y)+\overline{v_{h}}(y)\right)$ over the depth gives the bias voltage, which is fixed. Since resistivity is different, the depth profile is different at $200 \mathrm{~V}$, when A12A is not fully depleted. At $400 \mathrm{~V}$ both the sensors are fully depleted. Note that the full depths are slightly different between A07 and A12A. We conclude that the active depths are $10 \mu \mathrm{m}$ smaller than the physical thickness of the A07 and A12A samples, which are 310 and 320 $\mu \mathrm{m}$, respectively.

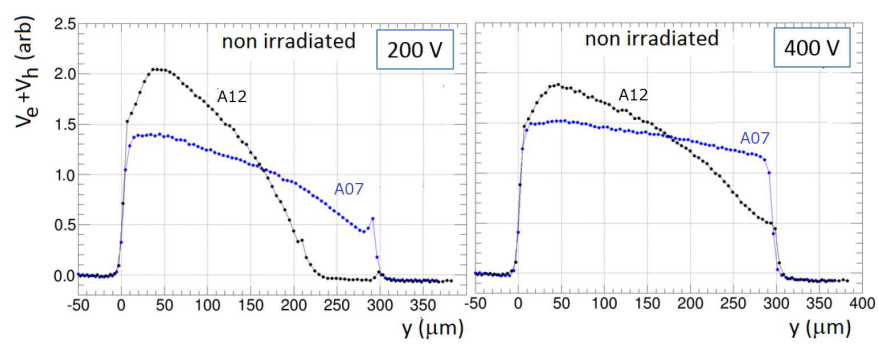

Fig. 11: Velocity-sum profiles of non-irradiated A07 and A12A samples at $200 \mathrm{~V}$ (left) and $400 \mathrm{~V}$ (right).

The velocity-sum profiles of neutron-irradiated A07 and A12A samples measured at $700 \mathrm{~V}$ are shown in Fig. 12. The field near the backside of A07 is substantially larger than that for A12A at $5 \times 10^{14} \mathrm{n}_{\mathrm{eq}} / \mathrm{cm}^{2}$ as expected, because of the different initial resistivities. The difference, however, diminishes at $2 \times 10^{15} \mathrm{n}_{\mathrm{eq}} / \mathrm{cm}^{2}$ irradiation, which can be explained by the fact that radiation-induced space charge dominates over the initial difference. Notably, non-zero velocity profiles near the backside are observed for both the samples at $2 \times 10^{15}$ $\mathrm{n}_{\mathrm{eq}} / \mathrm{cm}^{2}$ irradiation. Such a profile is known as "double peak electric field profile" [23] [22], where the space charge in the detector changes sign. The carriers released by the generation current are trapped in defects, and subsequently, holes flow to the backplane, effectively changing the sign of the space charge near the backplane of the p-type bulk. Therefore the space charge concentration becomes zero at a certain depth. For both the sensors, zero charge concentration occurs at a depth of approximately $180 \mu \mathrm{m}$ at $700 \mathrm{~V}$ at $2 \times 10^{15} \mathrm{n}_{\mathrm{eq}} / \mathrm{cm}^{2}$, and at approximately $250 \mu \mathrm{m}$ for A12A only at $5 \times 10^{14} \mathrm{n}_{\mathrm{eq}} / \mathrm{cm}^{2}$.

The velocity-sum profiles of protons-, neutron-, and pionirradiated A12A samples, measured at $300 \mathrm{~V}$ and $500 \mathrm{~V}$, are compared in Fig. 13. At both the bias voltages, there are substantial non-zero contributions near the backside for the pionirradiated samples. The profile reveals that the sample irradiated by pions to $5 \times 10^{14} \mathrm{n}_{\mathrm{eq}} / \mathrm{cm}^{2}$ is fully depleted at $500 \mathrm{~V}$, while there is a substantial undepleted region in the neutron-irradiated sample at $500 \mathrm{~V}$. The measured velocity-sum profiles agree with 


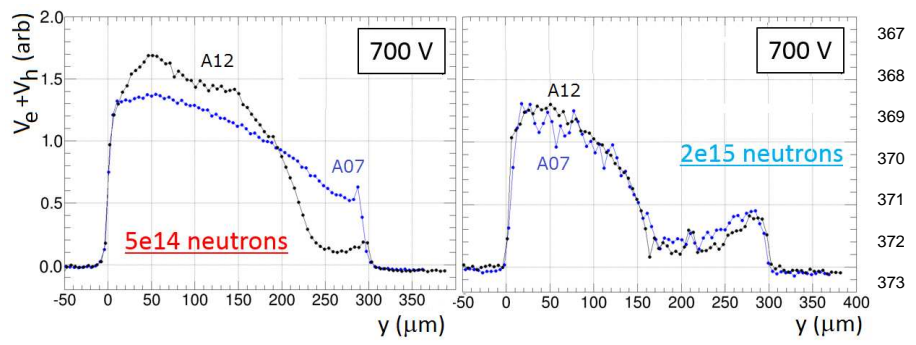

Fig. 12: Velocity-sum profiles of A07 and A12A samples at $700 \mathrm{~V}$ irradiated to ${ }^{374}$ a fluence of $5 \times 10^{14}$ (left) and $\mathrm{n}_{\mathrm{eq}} / \mathrm{cm}^{2} 2 \times 10^{15} \mathrm{n}_{\mathrm{eq}} / \mathrm{cm}^{2}$ (right).

the charge collection difference shown previously (Fig. 9).

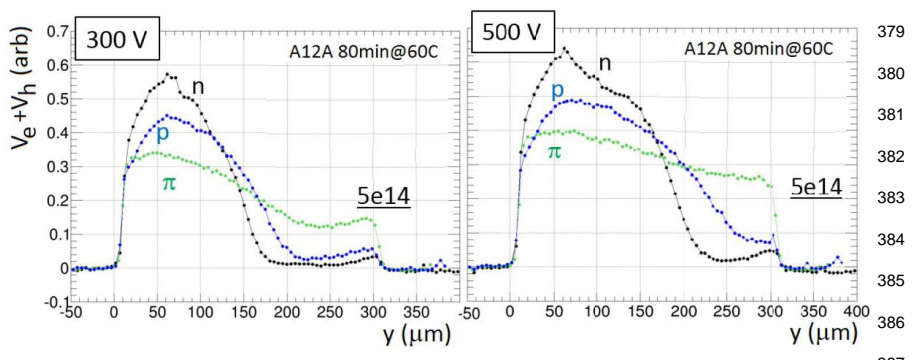

Fig. 13: Velocity-sum profiles of A12A samples irradiated with protons, neu- ${ }^{387}$ trons, and pions to a fluence of $5 \times 10^{14} \mathrm{n}_{\mathrm{eq}} / \mathrm{cm}^{2}$ measured at $300 \mathrm{~V}$ (left) and ${ }^{388}$ $500 \mathrm{~V}$ (right).

We extensively studied the charge collection from Hama ${ }^{394}$ matsu p-bulk FZ sensors of 310-320 $\mu$ m thickness using penetrating $\beta$ rays. Highly consistent results were obtained by the seven groups that participated in the measurements. The re- ${ }_{398}$ sults were further verified by independent measurements using ${ }_{39}$ an electron beam.

The difference in the charge collection between different sen- ${ }_{40.1}$ sor types, which is due to different initial resistivity, diminishes ${ }_{402}$ with the irradiation fluence and bias voltage. The effect depends on the particles used in the irradiation. For example, at $500 \mathrm{~V}$, the samples irradiated with protons showed a small difference in ${ }_{405}$ charge collection, while those irradiated with neutrons showed a difference below $1 \times 10^{15} \mathrm{n}_{\mathrm{eq}} / \mathrm{cm}^{2}$.

The reduction in the charge collection is largest for neu- ${ }^{407}$ tron irradiation followed by that for $23-\mathrm{MeV}$ proton irradiation. ${ }_{409}$ Damage caused by $300-\mathrm{MeV}$ pions is the least for the same $_{410}{ }_{40}$ NIEL fluence.

The carrier velocity profiles across the depth were evaluated using an edge TCT. The profiles differ for different irradiating particles, neutrons, protons, and pions. The field near the back-412 side is the largest for the pion-irradiated samples, which explains the largest charge collection observed for these samples. ${ }_{414}$

The expected signal-to-noise ratios after the HL-LHC fluence 415 can be evaluated using the ENC noise values of the readout ${ }^{416}$ electronics with corresponding wire-bonded sensors. Typical ${ }_{418}^{417}$ ENC noise values are $550 \mathrm{e}^{-}$for the barrel with $24 \mathrm{~mm} \mathrm{long}_{419}$ strips connected, $720 \mathrm{e}^{-}$for the barrel with $48 \mathrm{~mm}$ connected, ${ }^{420}$ and $650 \mathrm{e}^{-}$for the endcap module [24]. Assuming the safety factor of 2 and the neutron damage dominance, we arrive at conservative estimate for the lowest $\mathrm{S} / \mathrm{N}$ value of 14 . It is realized for endcap location.

The present studies have verified that the Hamamatsu p-bulk strip sensors are operational and provide precise particle tracking in the high-radiation environment expected in the HL-LHC.

\section{Acknowledgements}

The irradiations were performed: with protons at the University of Birmingham MC40 cyclotron, supported by the H2020 project AIDA-2020, GA number 654168, and the UK's Science and Technology Facilities Council, at Cyclotron and Radioisotope Center (CYRIC), Tohoku University, with Y.Sakemi, M. Ito, and T. Wakui, at the Karlsruhe Institute of Technology (KIT) by A. Dierlamm, supported by the Initiative and Networking Fund of the Helmholtz Association, contract HA-101 (Physics at the Terascale) and the European Commission under the FP7 Research Infrastructures project AIDA, Grant agreement no.262025, and at the LANSCE facility, Los Alamos National Laboratory; with neutrons at JSI TRIGA reactor in Ljubljana supported by the H2020 project AIDA-2020, GA no. 654168; with pions at Paul Scherrer Institut (PSI); and with $\gamma$ 's at Brookhaven National Laboratory (BNL).

The research was supported and financed in part by $\mathrm{CON}$ ICYT Becas Chile 72140349 and Cambridge Trust,, the Ministry of Education, Youth and Sports of the Czech Republic (Grant No. LG13009), the German Federal Ministry of Education and Research, and the Helmholtz Association, the European Social Fund and by the Ministry of Science, Research and Arts, Baden-Wuerttemberg, Germany, the Japan Society for Promoting Science KAKENHI-A Grant number 20244038 and KAKENHI-C Grant number 20540291, the Ministry of Education, Culture, Sports, Science and Technology-Japan, KAKENHI for Research on Priority Area Grant number 20025007 and for Scientific Research on Innovative Areas Grant number 23104002, the Slovenian Research Agency, the Spanish Ministry of Economy and Competitiveness through the Particle Physics National Program (ref. FPA2012-39055-C02-01 and FPA2012-39055-C02-02) and co-financed with FEDER funds, the financial support of the State Secretariat for Education, Research, and Innovation, the Swiss National Science Foundation and the Canton of Geneva, Switzerland, the UK Science and Technology Facilities Council (under Grant ST/M006409/1), and the United States Department of Energy, grant DE-FG0213ER41983.

\section{References}

[1] ATLAS Collaboration, JINST 3 (2008) S08003.

[2] S. McMahon, 2015. Presented at this symposium.

[3] M. Backhous, 2015. Presented at this symposium.

[4] I.-M. Gregor, 2015. Presented at this symposium.

[5] K. Hara and Y. Ikegami, Nuclear Instruments and Method A731 (2013) 242.

[6] ATLAS Collaboration, CERN-2012-022, LHCC-1-023 (2012).

[7] I. Dawson and P. Miyagawa, ATL-GEN-2014-003 (2014). 
[8] A. Vasilescu (INPE Bucharest) and G. Lindstroem (University of Hamburg), Displacement damage in silicon, on-line compilation, 2000. Http://rd50.web.cern.ch/RD50/NIEL/default.html.

[9] I. Dawson and P. Miyagawa (U. Sheffield), 2015 Aug. Private Communication.

[10] K. Hara, et al., Nuclear Instruments and Method A636 (2011) S83.

[11] Y. Unno, et al., Nuclear Instruments and Methods A636 (2011) S24.

[12] Y. Unno, et al., Nuclear Instruments and Method A765 (2014) 80.

[13] Alibava Systems, 2015. Homepage http://www.alibavasystems.com/.

[14] G. Kramberger, et al., IEEE TNS NS-57 (2010) 2294.

[15] L. Snoj,G. Žerovnik, and A. Trikov, Appl. Radiat. Isot. 70 (2012) 483.

[16] H. Bichsel, Review of Moder Physics 60-3 (1988) 663.

[17] G. Lindstroem, et al., Nuclear Instruments and Method A512 (2003) 30.

[18] I. Mandic, et al., Nuclear Instruments and Method A629 (2011) 101.

[19] M. Moll (ROSE Collaboration), Nuclear Instruments and Method A426 (1999) 87.

[20] RD50 Collaboration, CERN-LHCC-2010-012 and LHCC-SR-003 (2010).

[21] T. Affolder, Nuclear Instruments and Method A623 (2010) 177

[22] G. Kramberger, et al., JINST 9 (2014) P10016.

[23] V. Eremin, et al., Nuclear Instruments and Methods A535 (2004) 622.

[24] Communication with ATLAS ITk Strip Module group (T. Affolder et. al.), 2015 Sept. 